\title{
Nonpharmacological Interventions to Reduce Behavioral and Psychological Symptoms of Dementia: A Systematic Review
}

\author{
Alexandra Martini de Oliveira, ${ }^{1,2}$ Marcia Radanovic, ${ }^{1}$ Patrícia Cotting Homem de Mello, ${ }^{1,2}$ \\ Patrícia Cardoso Buchain, ${ }^{2}$ Adriana Dias Barbosa Vizzotto, ${ }^{2}$ \\ Diego L. Celestino, ${ }^{1}$ Florindo Stella, ${ }^{3}$ Catherine V. Piersol, ${ }^{4}$ and Orestes V. Forlenza ${ }^{1}$ \\ ${ }^{1}$ Laboratory of Neuroscience (LIM-27), Department and Institute of Psychiatry, Faculty of Medicine, University of São Paulo, \\ 05403-010 São Paulo, SP, Brazil \\ ${ }^{2}$ Occupational Therapy Service, Institute of Psychiatry, Faculty of Medicine, University of São Paulo, 05403-010 São Paulo, SP, Brazil \\ ${ }^{3}$ Biosciences Institute, Universidade Estadual Paulista (UNESP), 13506-900 Rio Claro, SP, Brazil \\ ${ }^{4}$ Department of Occupational Therapy, Clinical Director, Jefferson Elder Care, Thomas Jefferson University, Philadelphia, \\ PA 19107, USA
}

Correspondence should be addressed to Orestes V. Forlenza; forlenza@usp.br

Received 27 August 2015; Accepted 4 November 2015

Academic Editor: Hui X. Wang

Copyright (c) 2015 Alexandra Martini de Oliveira et al. This is an open access article distributed under the Creative Commons Attribution License, which permits unrestricted use, distribution, and reproduction in any medium, provided the original work is properly cited.

\begin{abstract}
Introduction. Behavioral and psychological symptoms of dementia (BPSD) are defined as a group of symptoms of disturbed perceptive thought content, mood, or behavior that include agitation, depression, apathy, repetitive questioning, psychosis, aggression, sleep problems, and wandering. Care of patients with BPSD involves pharmacological and nonpharmacological interventions. We reviewed studies of nonpharmacological interventions published in the last 10 years. Methods. We performed a systematic review in Medline and Embase databases, in the last 10 years, until June 2015. Key words used were (1) nonpharmacological interventions, (2) behavioral symptoms, (3) psychological symptoms, and (4) dementia. Results. We included 20 studies published in this period. Among these studies, program activities were more frequent (five studies) and the symptoms more responsive to the interventions were agitation. Discussion. Studies are heterogeneous in many aspects, including size sample, intervention, and instruments of measures. Conclusion. Nonpharmacological interventions are able to provide positive results in reducing symptoms of BPSD. Most studies have shown that these interventions have important and significant efficacy.
\end{abstract}

\section{Introduction}

The term BPSD stands for "behavioral and psychological symptoms of dementia" and has been used to describe a group of heterogeneous symptoms that arise in the course of dementia and are distressing and difficult to manage, both for caregivers and health professionals [1]. One or more symptoms of BPSD will affect up to $90 \%$ of patients with dementia during the disease course [2-4]. BPSD include many different behaviors such as screaming (disruptive vocalization), restlessness, repetitive questions, wandering, and apathy. As dementia is a progressive disease, BPSD worsen over time, requiring higher support and increasing cost of care [5]. BPSD have been associated with a poorer prognosis, a more rapid rate of cognitive decline, and illness progression [6], greater impairment in activities of daily living (ADLs) [6], and increased institutionalization at hospitals or residential care facilities $[2$, 7]. The frequency and severity of these symptoms have been strongly correlated with caregiver burden, reducing quality of life of patients and their caregivers [8].

Psychotropic medications have modest efficacy and can lead to undesired side effects [9-11] but they are frequently used to treat BPSD [12-15]. An increasing number of medical organizations and specialist groups such as the American Geriatric Society and the American Association for Geriatric Psychiatry currently consider nonpharmacological interventions to be first line Clinical Practice, except for emergency situations, referring mostly to situations in which the patient's 
behavior is harmful to him/her or other persons [16-18]. According to Cohen-Mansfield [19] most professionals have some training in medication prescription for BPSD, but few are instructed about nonpharmacological interventions or receive information about their effectiveness. As a consequence, antipsychotics drugs are frequently prescribed before alternative nonpharmacological approaches are attempted, and patients are maintained in medication for long periods, which leads to increasing morbidity and mortality. This scenario may be improved if professionals involved in dementia care are better apprised of the indications and limitations for the several existing nonpharmacological therapies for BPSD.

Some studies have shown that nonpharmacological treatments pose fewer side effects, which render them as safer options [20]. Nonpharmacological alternatives, including music therapy, aromatherapy, art therapy, behavioral therapy, reality orientation, tailored activities, and physical exercises, have shown promising results for the management of BPSD [21-23]. The aim of the present review was to identify and summarize the main nonpharmacological interventions for BPSD in the treatment of patients with dementia published in the last ten years.

\section{Methods}

2.1. Literature Review. We systematically searched the Medline and Embase databases using the following keywords: (1) non-pharmacological interventions, (2) behavioral symptoms, (3) psychological symptoms, and (4) dementia. These terms were selected even in the absence of specific Mesh terms as to increase sensitivity. The date limits ranged from the first paper published in 2005 to June 2015. We also looked for reviews to identify relevant articles about the issue.

2.2. Eligibility Criteria. To be included in the review, papers had to be written in English, Spanish, or Portuguese and have appropriate description of the study design (e.g., clinical trials, interventional studies, or clinical studies). Systematic reviews, meta-analyses, case reports, and editorial letters were not included in our review.

After selection and analysis of papers according to the above-mentioned inclusion criteria, the following variables were extracted and organized: (a) Overview: study design, authors, and year of publication; (b) Demographic: total sample (number of participants) and location; and (c) Assessment of BPSD. A critical analysis was performed in order to investigate the response of patients presenting diverse symptoms of BPSD to different nonpharmacological approaches.

\section{Results}

Our initial search returned 33 references. Of these, 20 studies met the inclusion criteria and were included in our review: five on activities, four on music therapy, three on aromatherapy, three on exercises, two on light therapy, one on touch therapy, one on combination of activities, and one on cognitive rehabilitation (Table 1). A brief comment and critical overview on selected studies is presented as follows.
3.1. Occupational Activities. The use of activities as nonpharmacological intervention for people with dementia has shown potential benefits in quality of life and in reducing agitation and depression [37-39]. Five studies investigated the effect of activities in BPSD in patients with dementia, with three addressing the "Tailored Activities Program" (TAP). TAP is an occupational therapy intervention program that focuses on reducing undesirable behaviors associated with dementia [28]. The principle of TAP is the selection of activities that are specifically tailored to the patient according to his/her abilities, interests, and roles. The program also offers training for caregivers in order to simplify activities and to adapt them for future functional declines of the patient as well as to generalize strategies to other contexts, thus helping caregivers to develop an increased sense of self-efficacy. In the US-TAP study, Gitlin et al. [28] demonstrated reduction in the overall incidence of BPSD and specific behaviors such as shadowing, agitation, argumentation, and repetitive questioning in a sample with 60 dyads. The US-TAP interventions proved to be effective in reducing shadowing $(p=0.003)$ and behavioral occurrences $(p=0.009)$. The Australian TAP was published as "protocol-only paper" and results are not yet available [11].

3.2. Music Therapy. Music therapy is one of the nonpharmacological methods used to reduce BPSD [26, 40]. We found four studies about the effectiveness of music therapy for the management of BPSD. One investigated the effects of two interventions, simulated family presence and preferred music, where participants were exposed to 15-minute audiotape sessions. One group of participants heard audiotapes with a conversation about positive experiences from the past and the other was exposed to a selection of songs that the individuals used to enjoy in their youth. Both interventions proved to be effective in reducing agitation [26].

Holmes et al. [24] compared two methods of presenting music, live or prerecorded, in the treatment of apathy. Music sessions comprised three different activities of 30 minutes each. One 30-minute session consisted of silence alone, another 30-minute session consisted of background prerecorded songs, and the last one consisted of watching live music sessions. Music played during the live interactive and prerecorded sessions was the same and consisted of a mixture of favorite songs according to the age of the group. Live interactive music proved to be more effective than prerecorded music in reducing apathy in moderate and severe dementia in the short term $(p<0.0001)$. Prerecorded music did not show any efficacy in improving apathy.

Sung et al. [33] investigated the effects of group music intervention on anxiety and agitation in institutionalized elders with dementia who actively participated in a music group session of 30 minutes, twice a week, for six weeks. The 30 minutes of intervention consisted of a five-minute warmup session with movements and breathing and a 20-minute session of active participation using percussion instruments and the last five minutes were a cool-down session with soft music. Previously the participants, caregivers, and family members were asked about patients' musical preferences and 


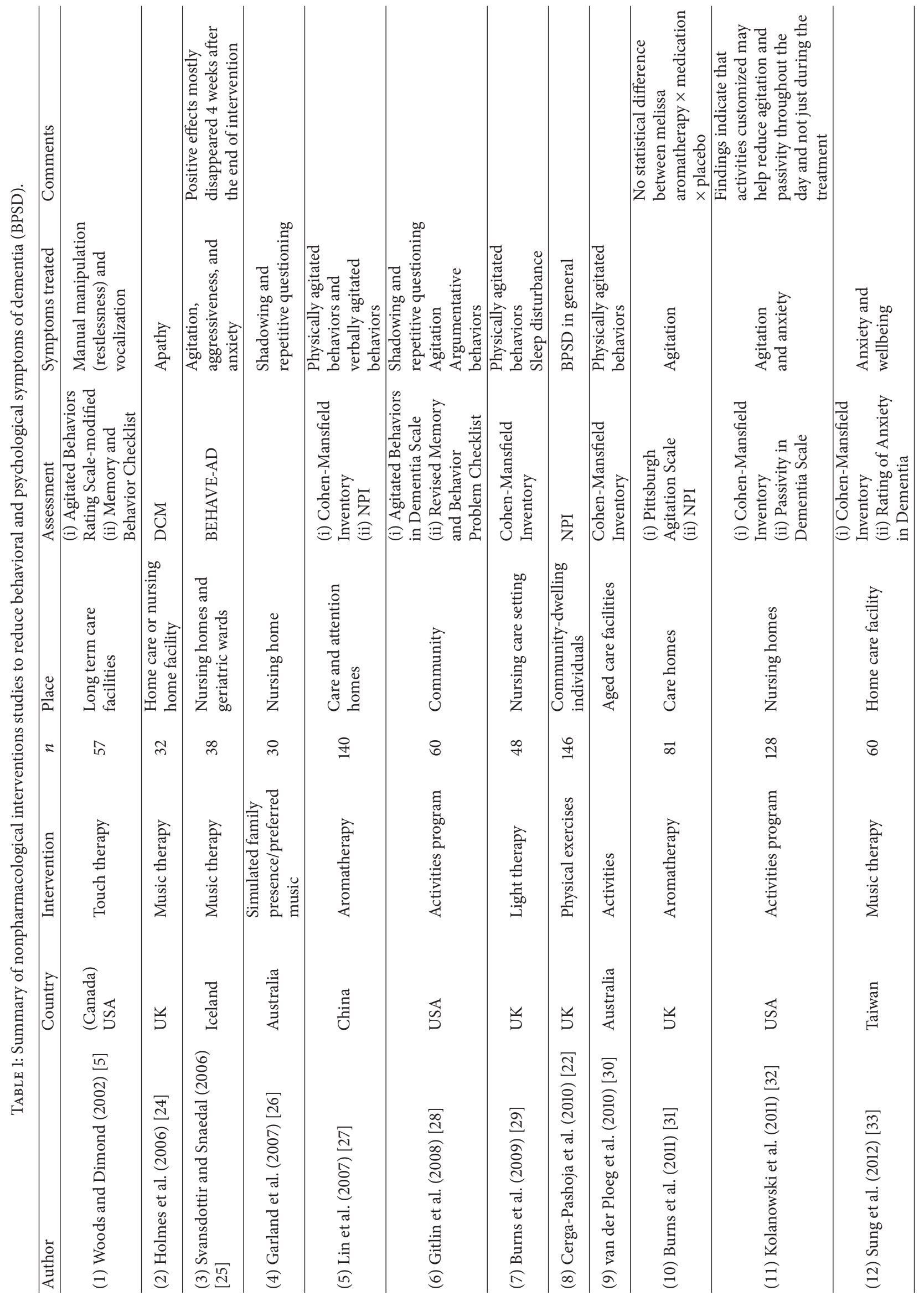




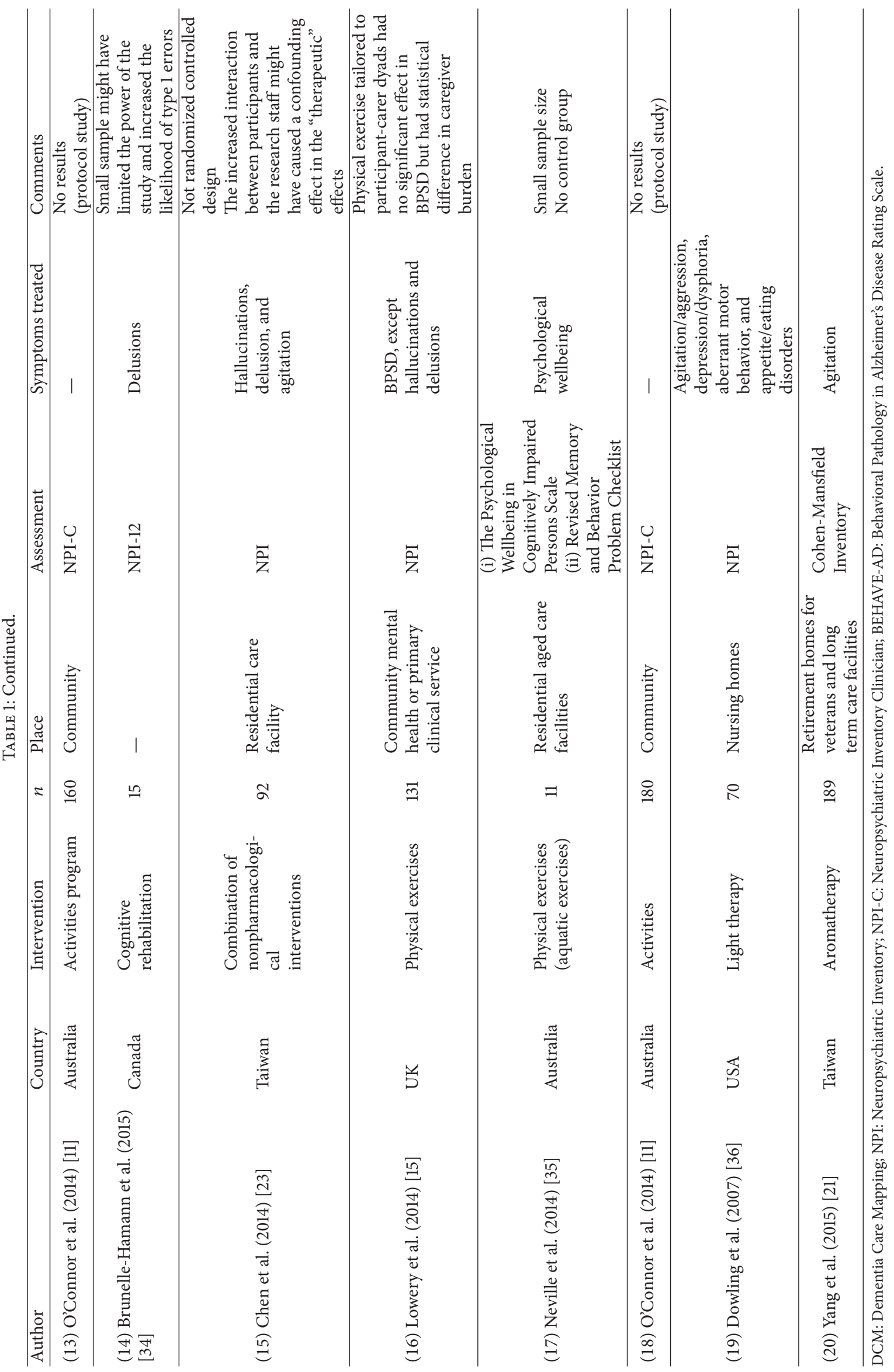


a selection of familiar songs was used in each session. Participants in the control group received routine care: activities of daily living, basic nursing care, meal provision, and some social activities (TV watching, family visiting, etc.). Results indicated that music intervention had a significant effect in reducing anxiety $(p=0.004)$.

Svansdottir and Snaedal [25] investigated the effect of music therapy in a case-control study in a sample of 38 patients and reported significant improvement in aggressiveness and anxiety. In this intervention patients and therapist sang songs chosen by the group: each song was sung twice, accompanied by a guitar and other instruments of their choice. The therapy group received 18 sessions of music therapy, each lasting 30 minutes, three times a week for 6 weeks, while the control group did not change their daily care routine. The authors concluded that music therapy significantly reduced agitation and anxiety $(p=0.02)$ in moderate and severe dementia.

3.3. Aromatherapy. Three studies examined the use of aromatherapy to treat BPSD. Aromatherapy involves the diffusion of aromatic oil into the environment. Two oils were used to treat agitation: lavender and melissa oil (lemon balm). Lin et al. [27] conducted a crossover randomized study, comparing lavender inhalation $(\mathrm{A})$, considered as the experimental group, and sunflower inhalation (B), considered as the placebo group. Lavender is a holistic relaxant that is regarded as having carminative, antiflatulent, and anticolic properties [27]. Sunflower preparation was selected as the placebo agent as it is odorless and does not possess any known therapeutic effect. Diffusers were placed on each side of the pillow of the participant during sleep at night for at least one hour. Each participant received both treatments (A and B) for three weeks with a washout period (two weeks) between each of the treatments. In this study, lavender was effective as an adjunctive therapy in alleviating agitation in patients with dementia $(p<0.001)$.

Burns et al. [31] assessed the efficacy of melissa aromatherapy in the treatment of agitation in dementia. This study was a randomized controlled trial, and the authors reported that there were no significant differences between aromatherapy, medication (donepezil), and placebo, with the three participant groups showing improvement in the NPI and in the Pittsburgh Agitation Scale (PAS).

Yang et al. [21] investigated aromatherapy and aroma acupressure (where acupuncture points were used in the aroma acupressure protocols to treat agitation). The procedures for the acupressure consisted of the following: each acupoint was pressed for two minutes with lavender oil and warm-up exercises were performed for five minutes, no longer than 15 minutes, once per day, five days a week, for four weeks in total. In the aromatherapy group, the lavender oil was applied at five acupoints with the same procedure. The aromatherapy and control groups did not receive any other interventions. Results showed that aroma-acupressure and aromatherapy had significant effect in reducing agitation $(p<0.01$ and $p=0.01$, resp.) when compared to the control group.
3.4. Physical Exercise. There were three articles on the effect of physical exercises on BPSD. However, one of them is just a "study protocol" without results [22]. From the remaining two studies, one addresses aquatic exercises, [35] consisting of a 45-minute group session (five to seven patients who performed exercises to strengthen agility, flexibility, and balance), followed by a relaxation session. Sessions were delivered twice a week over a 12 -week period with a trained instructor and some assistants. Each participant performed the exercises accompanied by an assistant. This study identified a significant decrease in the number of BPSD $(p=0.001)$, improvement in psychological wellbeing, and reduction in staff distress associated with BPSD $(p=0.001)$. The second study focused on the effect of physical exercises on BPSD, considering individually tailored walking regimen designed to become progressively intensive and to last between 20 and 30 minutes at least five times a week. Results showed that exercises did not improve BPSD but were effective in attenuating caregiver burden [15].

3.5. Bright Light Therapy. Bright light therapy (BLT) has been used with different results in patients with dementia, showing benefits in the management of agitation [35]. The most often reported positive results are improved night-time sleep, reduction in agitation, and improvement in cognitive performance. The study by Burns et al. [29] assessed the effect of BLT on BPSD and found that sleep quality was particularly improved in 48 patients with dementia. Their experimental group consisted of patients exposed to light with an intensity of 10,000 lux and the control group consisted of patients exposed to standard fluorescent tube light at 100 lux during two weeks for two hours per day. Agitation improved but there was no statistically significant effect.

Dowling et al. [36] tested the effects of BLT in a randomized trial, with the BLT being administered for one hour daily (Monday to Friday) for 11 weeks. One group was exposed to light in the morning period; the second group was exposed to light in the afternoon, and the third group was exposed to indoor light. There were statistically significant differences between morning light exposure and afternoon light exposure in agitation/aggression scores $(p=0.032)$ and between morning light and indoor light in aberrant motor behavior $(\mathrm{AMB})$ scores at the end of intervention $(p=0.021)$.

3.6. Touch Therapy. Touch therapies can include massage, craniosacral techniques, or therapeutic touch. Woods and Dimond [5] investigated the effect of touch therapy on BPSD in a double-blind three-group experimental study (one group with therapeutic touch, one group with placebo therapeutic touch, and the third group without any touch intervention). The interventions consisted of two daily sessions of 5-7 minutes for three days. The experimental group (therapeutic touch) experienced a statistically significant effect in reducing behavioral symptoms when compared to the group without any touch intervention $(p=0.036)$.

3.7. Combined Activities. One study investigated the efficacy of a combination of nonpharmacological interventions on 
BPSD among older Chinese men in Taiwan [23] in a prospective study with residents in dementia care units. The combination included music therapy, orientation training, physical exercise, and art cognitive activities. All the interventions were delivered by trained occupational therapists and the frequency of interventions for the study group was twice a week for 12 weeks. Music therapy consisted of activities encouraging participants to sing, to move their arms to the rhythm of the songs, and to use simple percussion instruments. Exercise included ball games and other recreational activities designed to increase the inhabitants' activity level. Art cognitive activities included various painting activities; for example, participants were asked to color a drawing of a beach scene and at the same time a conversation about the characteristics of summer was introduced to increase orientation. The intervention group had more significant reduction than the reference group in the NPI score $(p=0.046)$, including delusion $(p=0.018)$, hallucination $(p=0.004)$, and agitation $(p=0.038)[23]$.

3.8. Cognitive Rehabilitation. Only one study addressed cognitive rehabilitation for BPSD [34, 41]. The impact of cognitive interventions on the BPSD is still not well known because most of studies have focused on improving global or specific cognitive functions [41]. Considering the importance of the relationship between cognitive interventions and BPSD, Brunelle-Hamann et al. [34] evaluated a cognitive program in patients with mild and moderate $\mathrm{AD}$. This cognitive program consisted in a four-week home-based intervention of 45-60minute sessions twice a week for four weeks and involved memory techniques to relearn an instrumental activity of the daily living chosen by patients and caregivers (e.g., origami, computer, and TV remote control). The level of assistance was provided according to the necessity of each participant. After interventions, there was a significant reduction of delusional symptoms with a large effect size; however, aberrant motor behavior increased significantly in the treatment condition when compared to the control group [34].

\section{Discussion}

This paper provides an overview of current evidence on the efficacy of nonpharmacological interventions to reduce BPSD published in the last 10 years. All the interventions discussed in this review were dedicated to patients and caregivers. Studies are heterogeneous regarding intervention protocols, instruments of clinical assessment, and evaluation of outcome.

Five studies investigated the effectiveness of activity programs and demonstrated positive results. However, the intervention methods varied across studies. These studies used different theoretical backgrounds and investigated the effect of personalized activities. The largest effect size was found when the treatments were tailored to participants' interests and skills [30].

Four studies assessed the impact of music therapy, using different interventions, showing positive effects. The use of familiar songs reduced anxiety [24,33]. According to Gerdner
$[42,43]$, music can change the focus of attention and provide an interpretable stimulus that elicits positive memories from an earlier period in the person's life, which would prevent, or alleviate, anxiety or agitation. Live interactive music presents efficacy in the short term management of apathy in patients with moderate and severe dementia, whereas prerecorded music produces a more limited effect [24].

Researchers have investigated therapeutic touch with experimental and longitudinal study designs. Woods and Dimond [5] found that therapeutic touch can be used to decrease behavioral symptoms of dementia, specifically restlessness and vocalization. The mechanism of action of therapeutic touch is still unknown.

Bright light therapy (BLT) has been increasingly studied and regarded as appropriate method to improve fluctuations in diurnal rhythms that may account for night-time disturbances and the "sundown syndrome" (confusion or agitation in the late afternoon or early evening). The BLT studies included in this review revealed significant positive effects of this intervention in BPSD, especially in agitated behavior and sleep disturbance. The most likely explanation for these effects is the influence of BLT on the melatonin system, which is implicated in the regulation of abnormal motor behavior during sleep [31].

Aromatherapy may be beneficial to agitated patients with dementia [21, 27, 31]. However, varying degrees of anosmia have been reported in people with dementia [27], which might lead to analytical bias.

Most studies included in our review focused on and reported behavioral abnormalities such as agitation. However, one study showed that an organized nonpharmacological intervention program was effective in managing both outward and intrinsic symptoms, including hallucination and delusion [23].

Studies focusing on the implementation of physical exercises programs have demonstrated reduction in BPSD and improvement of psychological wellbeing in patients with dementia. However, most studies were based on small samples, and further studies are warranted.

In our review, ten of the twenty studies indicated that nonpharmacological interventions are effective in reducing agitation. Agitation is a very common, persistent, and distressing symptom among people with moderate and severe dementia, affecting 30\% of those living at home [44]. According to Livingston et al. [45], agitation in dementia is associated with poorer quality of life and impairs the engagement in daily activities and relationships. In addition to causing distress in family members and caregivers, it may precipitate institutionalization at nursing homes.

Currently, in clinical practice, pharmacological treatment of agitation is usually performed using antipsychotic drugs. However, clinical outcomes are poor and undesired side effects (including cognitive worsening, confusion, and extrapyramidal signs) are frequent, even with the use of the newer atypical drugs [14]. Therefore, nonpharmacological interventions seem to provide safer and effective alternatives for treating agitation in patients with dementia.

Regarding clinical settings, the majority of the studies ( $n=15)$ included in this review focused on interventions 
on patients with dementia residing at long term care facilities, and their application to home-based support remains uncertain [46, 47]. According to Trivedi et al. [1], two-thirds of patients with dementia live at home and yet there is limited evidence on which methods are the most effective in this setting.

Interestingly, one study reported significant worsening on BPSD. Brunelle-Hamann et al. [34], in a single blind, blockrandomized and crossover-controlled study, investigated the impact of cognitive rehabilitation program on BPSD in AD patients. The results revealed that aberrant motor behaviors increased significantly in the treatment condition when compared to the control condition. The proposed hypothesis was that, during the rehabilitation intervention, as the dementia progresses, AD patients gradually lose their coping abilities and perceive their environment as more stressful.

Some limitations of the included studies need to be addressed. In terms of BPSD measures, most scales rely on information provided by caregivers, being thus subjected to the interference of variables such as caregiver's burden, personality, and even his/her ability to perceive changes in patients' behavior. However, the studies addressed in our review employed instruments that are validated and widely used in dementia research. Our review encompassed singleblind, double-blind, case-control, and prospective studies. Although these studies are heterogeneous in terms of design, intervention methods, and measures of outcome, bias can be reduced using statistical analysis strategies.

Some studies included in our review address tailored interventions [11, 26, 39]. According to Cohen-Mansfield [19], dementia patients become agitated when their needs are not perceived or addressed by caregivers. These needs can be addressed by a "person-centered care model." Tailored interventions are currently being considered as more effective than standardized interventions. Garland et al. [26] reported that audiotapes containing a family member's voice were more effective than audiotapes with songs to reduce agitation in patients with BPSD. Gitlin et al. [48] and O'Connor et al. [11] described a home-based occupational therapy program based on personal capabilities and individual preferences. This tailored program promoted a significantly greater reduction in agitation. In a randomized controlled trial, Gitlin et al. [48] found that nonpharmacological interventions based on Tailored Activities Program are cost-effective and should be considered as part of the clinical management of dementia.

\section{Conclusion}

Studies focusing on alternative approaches have disclosed that different nonpharmacological interventions are able to provide positive results in reducing symptoms of BPSD. Most studies have demonstrated that these interventions have important and significant efficacy improving BPSD such as agitation, psychotic symptoms, and apathy. Undesired side effects of pharmacological treatments, as antipsychotics and benzodiazepines, have promoted a search for alternative treatments for BPSD. Therefore, nonpharmacological interventions programs should be considered as first-option interventions to treat BPSD.

\section{Conflict of Interests}

The authors declare that there is no conflict of interests regarding the publication of this paper.

\section{Acknowledgments}

Fundação de Amparo à Pesquisa do Estado de São Paulo (FAPESP) (Grant no. 09/52825-8, Brazil), Associação Beneficente Alzira Denise Hertzog da Silva (ABADHS), and JNK Empreendimentos e Incorporações financially supported this work.

\section{References}

[1] D. Trivedi, C. Goodman, A. Dickinson et al., "A protocol for a systematic review of research on managing behavioural and psychological symptoms in dementia for community-dwelling older people: evidence mapping and syntheses," Systematic Reviews, vol. 2, article 70, 2013.

[2] H. C. Kales, H. M. Kim, K. Zivin et al., "Risk of mortality among individual antipsychotics in patients with dementia," The American Journal of Psychiatry, vol. 169, no. 1, pp. 71-79, 2012.

[3] C. G. Lyketsos, O. Lopez, B. Jones, A. L. Fitzpatrick, J. Breitner, and S. DeKosky, "Prevalence of neuropsychiatric symptoms in dementia and mild cognitive impairment: results from the cardiovascular health study," The Journal of the American Medical Association, vol. 288, no. 12, pp. 1475-1483, 2002.

[4] E. C. Hersch and S. Falzgraf, "Management of the behavioral and psychological symptoms of dementia," Clinical Interventions in Aging, vol. 2, no. 4, pp. 611-621, 2007.

[5] D. L. Woods and M. Dimond, "The effect of therapeutic touch on agitated behavior and cortisol in persons with Alzheimer's disease," Biological Research for Nursing, vol. 4, no. 2, pp. 104$114,2002$.

[6] J. S. Paulsen, D. P. Salmon, L. J. Thal et al., "Incidence of and risk factors for hallucinations and delusions in patients with probable AD," Neurology, vol. 54, no. 10, pp. 1965-1971, 2000.

[7] M. Haupt, A. Kurz, and M. Jänner, "A 2-year follow-up of behavioural and psychological symptoms in Alzheimer's disease," Dementia and Geriatric Cognitive Disorders, vol. 11, no. 3, pp. 147-152, 2000.

[8] S. Finkel, "Introduction to behavioural and psychological symptoms of dementia (BPSD)," International Journal of Geriatric Psychiatry, vol. 15, supplement 1, pp. S2-S4, 2000.

[9] L. S. Schneider, K. Dagerman, and P. S. Insel, "Efficacy and adverse effects of atypical antipsychotics for dementia: metaanalysis of randomized, placebo-controlled trials," American Journal of Geriatric Psychiatry, vol. 14, no. 3, pp. 191-210, 2006.

[10] N. Kar, "Behavioral and psychological symptoms of dementia and their management," Indian Journal of Psychiatry, vol. 51, supplement 1, pp. S77-S86, 2009.

[11] C. M. O'Connor, L. Clemson, H. Brodaty, Y. H. Jeon, E. Mioshi, and L. N. Gitlin, "Use of the Tailored Activities Program to reduce neuropsychiatric behaviors in dementia: an Australian protocol for a randomized trial to evaluate its effectiveness," International Psychogeriatrics, vol. 26, no. 5, pp. 857-869, 2014.

[12] H. C. Kales, M. Valenstein, H. M. Kim et al., "Mortality risk in patients with dementia treated with antipsychotics versus other psychiatric medications," The American Journal of Psychiatry, vol. 164, no. 10, pp. 1568-1576, 2007. 
[13] C. Ballard, I. Ziabreva, R. Perry et al., "Differences in neuropathologic characteristics across the Lewy body dementia spectrum," Neurology, vol. 67, no. 11, pp. 1931-1934, 2006.

[14] C. Ballard, M. L. Hanney, M. Theodoulou et al., "The dementia antipsychotic withdrawal trial (DART-AD): long-term followup of a randomised placebo-controlled trial," The Lancet Neurology, vol. 8, no. 2, pp. 151-157, 2009.

[15] D. Lowery, A. Cerga-Pashoja, S. Iliffe et al., "The effect of exercise on behavioural and psychological symptoms of dementia: the EVIDEM-E randomised controlled clinical trial," International Journal of Geriatric Psychiatry, vol. 29, no. 8, pp. 819-827, 2014.

[16] American Psychiatric Association, Diagnostic and Statistical Manual of Mental Disorders, American Psychiatric Publishing, Arlington, Va, USA, 2013.

[17] Royal College of Nursing, Dementia: Supporting People with Dementia and Their Careers in Health and Social Care, National Institute for Health and Clinical Excellence, London, UK, 2006, http://www.scie.org.uk/publications/misc/dementia/dementiaguideline.pdf?res=true.

[18] American Geriatrics Society, Choosing Wisely, An Initiative of the ABIM Foundation. Five Things Physicians and Patients Should Question, American Geriatrics Society, 2013.

[19] J. Cohen-Mansfield, "Nonpharmacologic interventions for inappropriate behaviors in dementia: a review, summary, and critique," The American Journal of Geriatric Psychiatry, vol. 9, no. 4, pp. 361-381, 2001.

[20] S. S. Gill, S. E. Bronskill, S.-L. T. Normand et al., "Antipsychotic drug use and mortality in older adults with dementia," Annals of Internal Medicine, vol. 146, no. 11, pp. 775-786, 2007.

[21] M. H. Yang, L. C. Lin, S. C. Wu, J. H. Chiu, P. N. Wang, and J. G. Lin, "Comparison of the efficacy of aroma-acupressure and aromatherapy for the treatment of dementia-associated agitation," BMC Complementary and Alternative Medicine, vol. 15, article 93, 2015.

[22] A. Cerga-Pashoja, D. Lowery, R. Bhattacharya et al., "Evaluation of exercise on individuals with dementia and their carers: a randomised controlled trial," Trials, vol. 11, article 53, 2010.

[23] R.-C. Chen, C.-L. Liu, M.-H. Lin et al., "Non-pharmacological treatment reducing not only behavioral symptoms, but also psychotic symptoms of older adults with dementia: a prospective cohort study in Taiwan," Geriatrics and Gerontology International, vol. 14, no. 2, pp. 440-446, 2014.

[24] C. Holmes, A. Knights, C. Dean, S. Hodkinson, and V. Hopkins, "Keep music live: music and the alleviation of apathy in dementia subjects," International Psychogeriatrics, vol. 18, no. 4, pp. 623-630, 2006.

[25] H. B. Svansdottir and J. Snaedal, "Music therapy in moderate and severe dementia of Alzheimer's type: a case-control study," International Psychogeriatrics, vol. 18, no. 4, pp. 613-621, 2006.

[26] K. Garland, E. Beer, B. Eppingstall, and D. W. O'Connor, "A comparison of two treatments of agitated behavior in nursing home residents with dementia: simulated family presence and preferred music," The American Journal of Geriatric Psychiatry, vol. 15, no. 6, pp. 514-521, 2007.

[27] P. W.-K. Lin, W.-C. Chan, B. F.-L. Ng, and L. C.-W. Lam, "Efficacy of aromatherapy (Lavandula angustifolia) as an intervention for agitated behaviours in Chinese older persons with dementia: a cross-over randomized trial," International Journal of Geriatric Psychiatry, vol. 22, no. 5, pp. 405-410, 2007.

[28] L. N. Gitlin, L. Winter, J. Burke, N. Chemett, M. P. Dennis, and W. W. Hauck, “Tailored activities to manage neuropsychiatric behaviors in persons with dementia and reduce caregiver burden: a randomized pilot study," The American Journal of Geriatric Psychiatry, vol. 16, no. 3, pp. 229-239, 2008.

[29] A. Burns, H. Allen, B. Tomenson, D. Duignan, and J. Byrne, "Bright light therapy for agitation in dementia: a randomized controlled trial," International Psychogeriatrics, vol. 21, no. 4, pp. 711-721, 2009.

[30] E. S. van der Ploeg, B. Eppingstall, and D. W. O'Connor, “The study protocol of a blinded randomised-controlled cross-over trial of lavender oil as a treatment of behavioural symptoms in dementia," BMC Geriatrics, vol. 10, no. 1, article 49, 2010.

[31] A. Burns, E. Perry, C. Holmes et al., "A double-blind placebocontrolled randomized trial of Melissa officinalis oil and donepezil for the treatment of agitation in Alzheimer's disease," Dementia and Geriatric Cognitive Disorders, vol. 31, no. 2, pp. 158-164, 2011.

[32] A. Kolanowski, M. Litaker, L. Buettner, J. Moeller, and P. T. Costa Jr., "A randomized clinical trial of theory-based activities for the behavioral symptoms of dementia in nursing home residents," Journal of the American Geriatrics Society, vol. 59, no. 6, pp. 1032-1041, 2011.

[33] H.-C. Sung, W.-L. Lee, T.-L. Li, and R. Watson, "A group music intervention using percussion instruments with familiar music to reduce anxiety and agitation of institutionalized older adults with dementia," International Journal of Geriatric Psychiatry, vol. 27, no. 6, pp. 621-627, 2012.

[34] L. Brunelle-Hamann, S. Thivierge, and M. Simard, "Impact of a cognitive rehabilitation intervention on neuropsychiatric symptoms in mild to moderate Alzheimer's disease," Neuropsychological Rehabilitation, vol. 25, no. 5, pp. 677-707, 2015.

[35] C. Neville, T. Henwood, E. Beattie, and E. Fielding, "Exploring the effect of aquatic exercise on behaviour and psychological well-being in people with moderate to severe dementia: a pilot study of the Watermemories Swimming Club," Australasian Journal on Ageing, vol. 33, no. 2, pp. 124-127, 2014.

[36] G. A. Dowling, C. L. Graf, E. M. Hubbard, and J. S. Luxenberg, "Light treatment for neuropsychiatric behaviors in Alzheimer's disease," Western Journal of Nursing Research, vol. 29, no. 8, pp. 961-975, 2007.

[37] M. J. L. Graff, M. J. M. Vernooij-Dassen, M. Thijssen, J. Dekker, W. H. L. Hoefnagels, and M. G. M. O. Rikkert, "Community based occupational therapy for patients with dementia and their care givers: randomised controlled trial," British Medical Journal, vol. 333, no. 7580, pp. 1196-1199, 2006.

[38] D. J. Brooker, R. J. Woolley, and D. Lee, "Enriching opportunities for people living with dementia in nursing homes: an evaluation of a multi-level activity-based model of care," Aging and Mental Health, vol. 11, no. 4, pp. 361-370, 2007.

[39] J. Fraker, H. C. Kales, M. Blazek, J. Kavanagh, and L. N. Gitlin, "The role of the occupational therapist in the management of neuropsychiatric symptoms of dementia in clinical settings," Occupational Therapy in Health Care, vol. 28, no. 1, pp. 4-20, 2014.

[40] L. A. Gerdner, "Individualized music for dementia: evolution and application of evidence-based protocol," World Journal of Psychiatry, vol. 2, no. 2, pp. 26-32, 2012.

[41] A. Bahar-Fuchs, L. Clare, and B. Woods, "Cognitive training and cognitive rehabilitation for persons with mild to moderate dementia of the Alzheimer's or vascular type: a review," Alzheimer's Research and Therapy, vol. 5, no. 4, article 35, 2013. 
[42] L. A. Gerdner, "Music, art, and recreational therapies in the treatment of behavioral and psychological symptoms of dementia," International Psychogeriatrics, vol. 12, no. 1, pp. 359-366, 2000.

[43] L. A. Gerdner, "Effects of individualized versus classical 'relaxation' music on the frequency of agitation in elderly persons with Alzheimer's disease and related disorders," International Psychogeriatrics, vol. 12, no. 1, pp. 49-65, 2000.

[44] S.-H. Ryu, C. Katona, B. Rive, and G. Livingston, "Persistence of and changes in neuropsychiatric symptoms in Alzheimer disease over 6 months: the LASER-AD study," The American Journal of Geriatric Psychiatry, vol. 13, no. 11, pp. 976-983, 2005.

[45] G. Livingston, K. Johnston, C. Katona, J. Paton, and C. G. Lyketsos, "Systematic review of psychological approaches to the management of neuropsychiatric symptoms of dementia," The American Journal of Psychiatry, vol. 162, no. 11, pp. 1996-2021, 2005.

[46] D. W. O'Connor, D. Ames, B. Gardner, and M. King, "Psychosocial treatments of behavior symptoms in dementia: a systematic review of reports meeting quality standards," International Psychogeriatrics, vol. 21, no. 2, pp. 225-240, 2009.

[47] D. W. O'Connor, D. Ames, B. Gardner, and M. King, "Psychosocial treatments of psychological symptoms in dementia: a systematic review of reports meeting quality standards," International Psychogeriatrics, vol. 21, no. 2, pp. 241-251, 2009.

[48] L. N. Gitlin, N. Hodgson, E. Jutkowitz, and L. Pizzi, "The costeffectiveness of a nonpharmacologic intervention for individuals with dementia and family caregivers: the tailored activity program," The American Journal of Geriatric Psychiatry, vol. 18, no. 6 , pp. 510-519, 2010. 


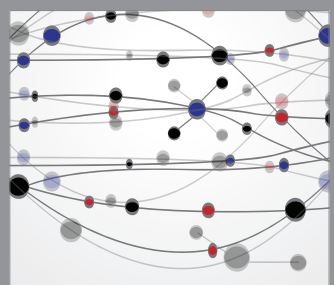

The Scientific World Journal
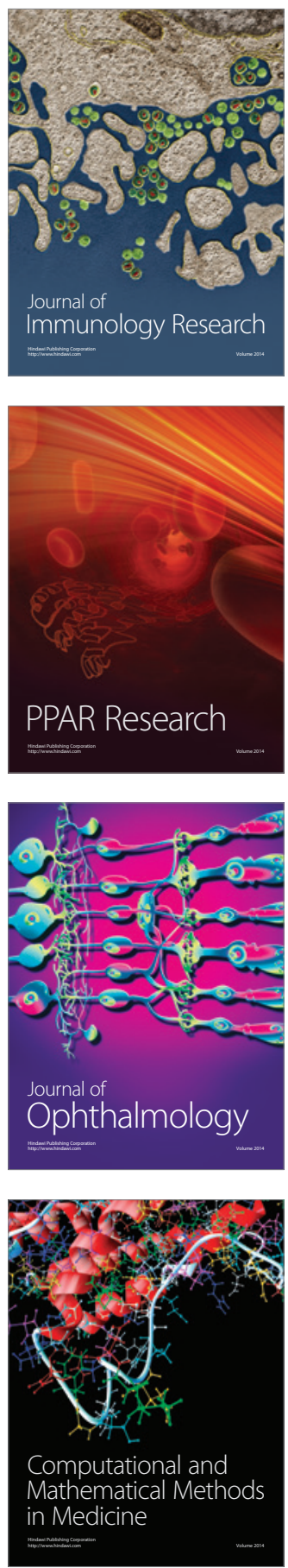

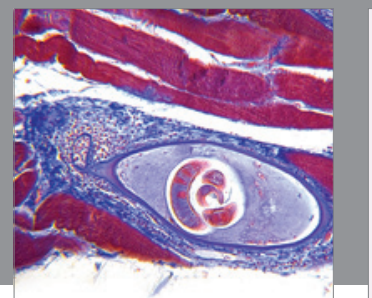

Gastroenterology

Research and Practice
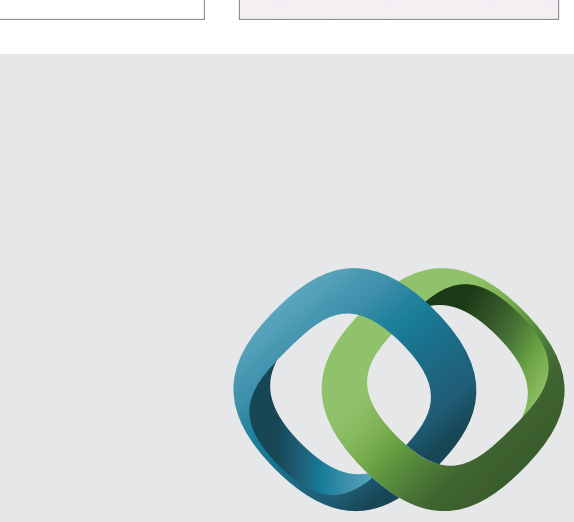

\section{Hindawi}

Submit your manuscripts at

http://www.hindawi.com
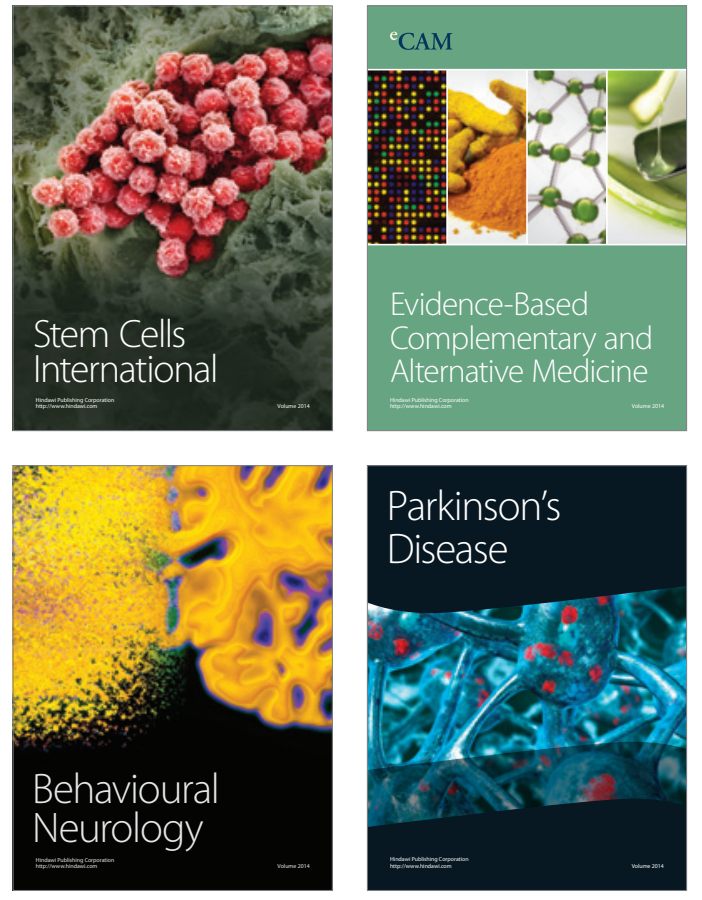
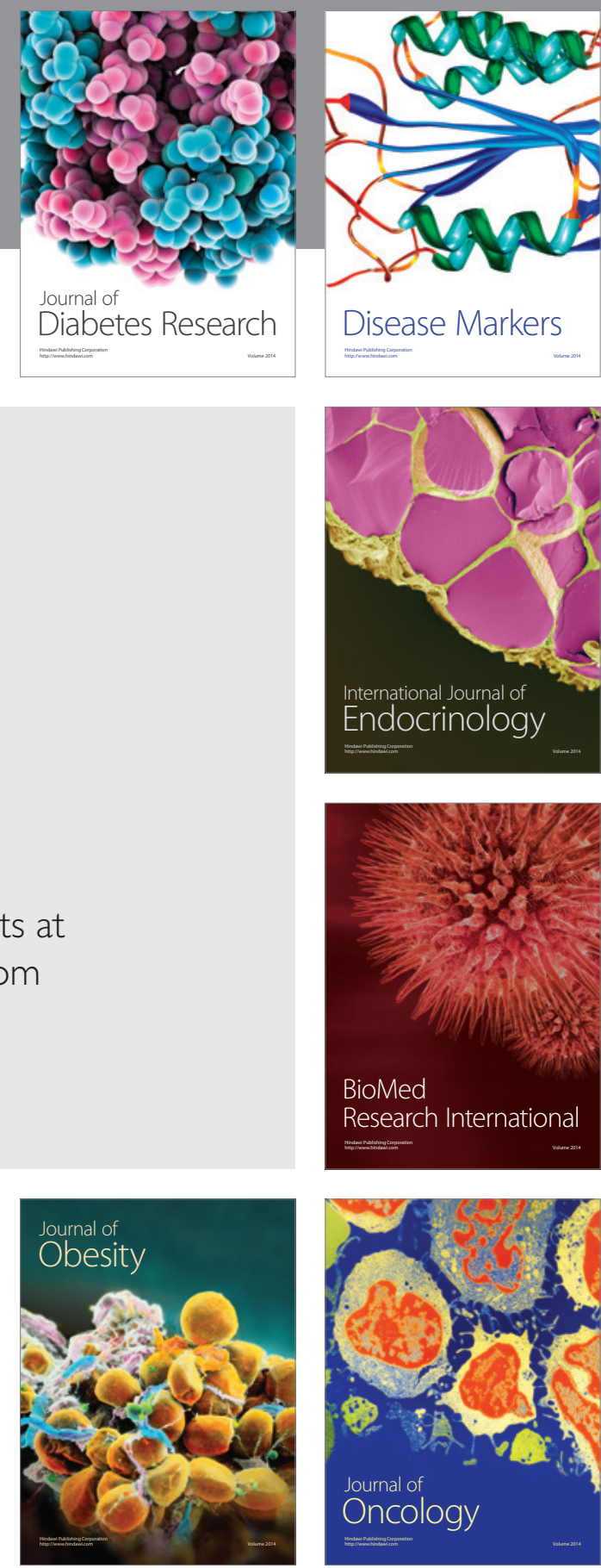

Disease Markers
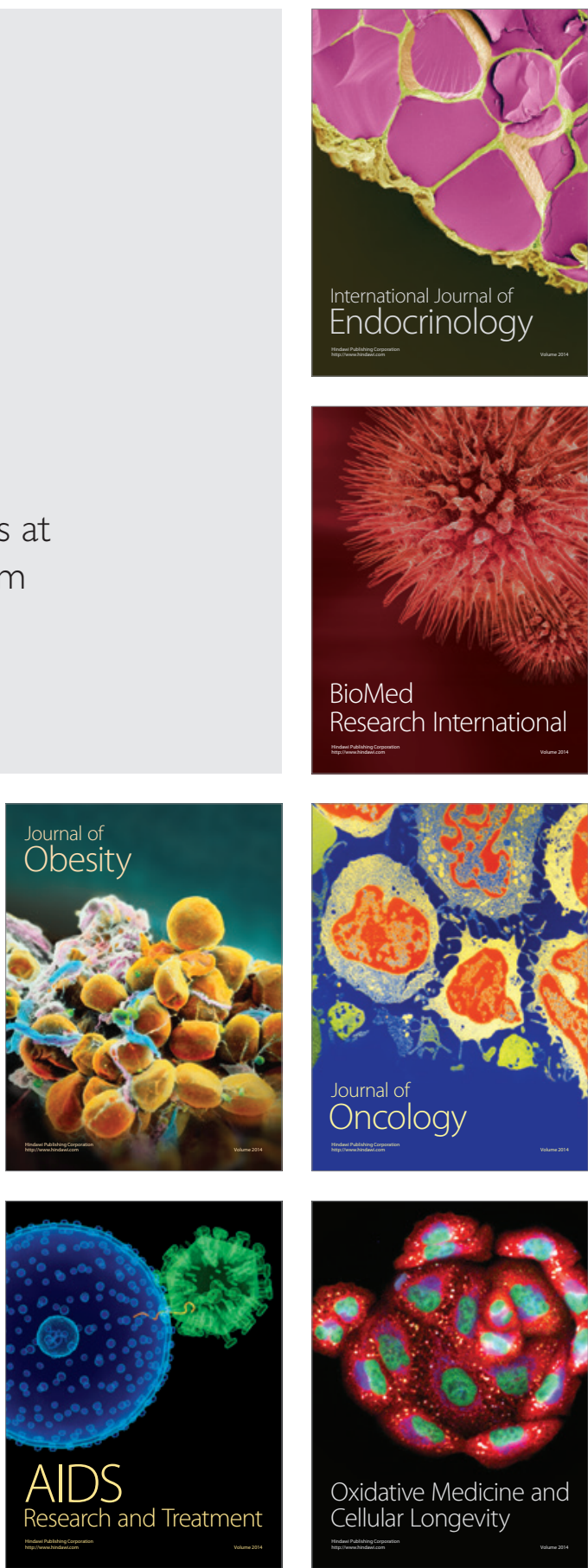